> Initialement développée pour le traitement de pathologies neurologiques, comme la maladie de Parkinson, la stimulation cérébrale profonde est actuellement utilisée dans certaines pathologies psychiatriques telles que les troubles obsessionnels compulsifs ou la dépression. Elle commence à être envisagée, voire appliquée, comme traitement de certaines addictions. Nous faisons le point dans cette revue sur l'évolution des discussions sur l'intérêt de cette approche thérapeutique, et discutons en particulier les arguments expérimentaux orientant le choix vers les deux cibles cérébrales les plus pertinentes pour traiter les addictions par stimulation cérébrale profonde: le noyau accumbens (NAc) et le noyau sous-thalamique (NST). <

La dépendance à une drogue est une pathologie psychiatrique qui, pendant longtemps, n'a pas été considérée comme telle. En effet, l'addiction ne se résume pas au simple usage de drogue mais bien à une perte du contrôle de sa consommation et à une recherche compulsive et obsessionnelle de la substance. Elle est l'aboutissement d'une progression plus ou moins lente d'une consommation récréationnelle initiale, à une consommation abusive et enfin toxicomaniaque. Les travaux de recherche menés sur les conduites addictives ont montré que tous les individus ne sont pas égaux face à ce phénomène. Ainsi, alors que la plupart des usagers récréationnels maintiennent ce comportement pendant de longues périodes, d'autres basculent rapidement dans un état de consommation abusif, puis dans l'addiction. L'enjeu des recherches actuelles est de mieux identifier les facteurs à l'origine de ces différences de comportement $[43,44]$.

Comment peut-on venir en aide aux toxicomanes actuellement? Beaucoup de personnes continuent de penser que sortir d'une dépendance n'est qu'une ques-

Cet article fait partie de la série «Addictions » dont la parution a débuté dans le $n^{\circ} 4$, vol. 31, avril 2015 (www.medecinesciences.org).

\section{Addictions (4) \\ La chirurgie \\ au secours \\ des addictions}

Yann Pelloux, Christelle Baunez

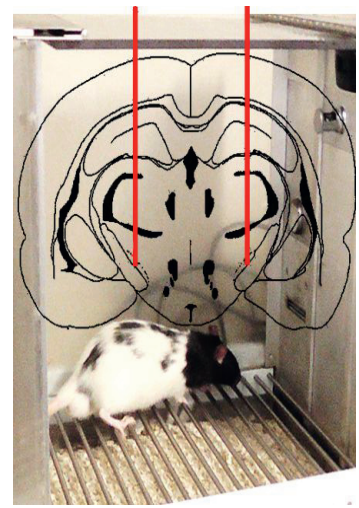

Équipe BAGAMORE (ganglions de la base, motivation et récompense), institut de neurosciences de la Timone, UMR7289 CNRS et Aix-Marseille université, campus santé Timone, 27, boulevard Jean Moulin, 13385 Marseille cedex 05, France.

christelle.baunez@univ-amu.fr

tion de volonté. Cependant, certaines formes de dépendances peuvent être atténuées, voire stoppées, grâce à des substituts de la drogue (comme la méthadone utilisée dans les cas de dépendance aux opiacés). De même, les thérapies comportementales peuvent contribuer à un arrêt de la conduite toxicomaniaque. Dans le cas de l'alcool par exemple, une démarche actuellement débattue consiste à proposer à certains alcooliques non pas une attitude d'abstinence complète telle que prônée jusqu'à présent, mais une attitude de consommation contrôlée. L'abstinence complète était jusqu'à présent principalement recommandée en partant du principe qu'un individu addict restera vulnérable toute sa vie, et qu'il n'existe aucun traitement satisfaisant pour l'aider à maintenir une consommation contrôlée.

Les progrès de la neurochirurgie appliquée aux maladies neurologiques comme la maladie de Parkinson, ont également permis un renouveau des approches chirurgicales appliquées aux maladies psychiatriques. Celles-ci étaient en effet devenues «tabou» à la suite des effets secondaires désastreux des lobotomies et autres chirurgies appliquées à la psychiatrie. Mais le développement de la stimulation cérébrale profonde (SCP) - qui a été utilisée récemment chez des patients souffrant de dépression sévère et résistante aux médicaments, mais également chez des patients souffrant de troubles obsessionnels compulsifs (TOC) [45] - a relancé la discussion sur l'intérêt d'une thérapeutique chirurgicale dans les addictions.

\section{Qu'est-ce que la stimulation cérébrale profonde?}

La stimulation cérébrale profonde consiste en l'implantation d'une électrode dans une zone cérébrale déterminée et en l'application d'un courant, souvent à haute fréquence $(130 \mathrm{~Hz})$, délivré au moyen d'un générateur implanté chez le patient, au niveau de la clavicule (comme 
illustré sur la Figure 1). Appliquée chez le patient parkinsonien dans une région du cerveau alors hyperactive (par ex. le noyau subthalamique), elle est sensée avoir un effet d'inactivation : les symptômes moteurs parkinsoniens disparaissent en effet lors de l'activation de la stimulation à haute fréquence au niveau de cette zone. Cela a été démontré pour la première fois en France par l'équipe du Pr Benabid à Grenoble [1]. Les mécanismes d'action sont en fait plus complexes, mais le consensus actuel est de considérer que le processus repose sur l'inactivation de la structure ciblée et la stimulation des fibres passant à proximité du site.

Alors que la stratégie d'inactivation d'une zone cérébrale a longtemps consisté à produire des lésions (consistant en des destructions irréversibles), l'avantage de la stimulation cérébrale profonde est d'être adaptable (il est possible de choisir le point de stimulation le plus efficace et d'ajuster l'intensité du courant) et réversible (la stimulation peut à tout moment être arrêtée en cas d'intolérance, et les électrodes retirées si nécessaire).

\section{Dans quelles régions cérébrales appliquer la stimulation profonde pour traiter les addictions?}

À partir d'observations faites chez des patients, ou sur la base d'études réalisées chez l'animal, deux régions cérébrales semblent les cibles les plus pertinentes actuellement pour traiter les addictions par stimulation cérébrale profonde: le noyau accumbens (NAc) et le noyau sous-thalamique (NST) (Figure 2).

\section{La stimulation profonde du noyau accumbens}

L'application clinique de la stimulation profonde dans le traitement de la dépendance chez l'homme s'est jusqu'à présent principalement focalisée sur le noyau accumbens (NAc). Le NAc (ou striatum ventral) est une structure des ganglions de la base ${ }^{1}$ qui est considérée comme étant au centre du système de renforcement avec, entre autres, le renforcement par les drogues [44] $(\rightarrow)$.

$\rightarrow$ Pour une description du rôle du NAc dans l'addiction, voir la Synthèse de M. Arrango-Lievano et M.G. Kaplitt, $\mathrm{m} / \mathrm{s}^{\circ}{ }^{\circ}$, mai 2015, page 546

Nous soutenons néanmoins que le choix du NAc comme structure cible de la stimulation profonde dans le traitement de la dépendance aux drogues se fonde sur des bases théoriques très controversées, qui présupposent une participation préférentielle du NAc dans les comportements toxicomaniaques. Nous nous fondons sur deux arguments : 1. Il est important de noter qu'il n'existe aucune preuve clairement établie d'une altération spécifique du NAc chez les personnes toxicomanes. En fait, on retrouve chez les toxicomanes une augmentation de la matière grise à la fois dans le striatum ventral et le striatum dorsal [2]. Ces résultats sont en accord avec des études précliniques réalisées chez le singe, qui montrent que les modifications observées initialement au niveau du striatum ventral, dans une situation où l'usage de drogue est toujours récréatif, atteignent le striatum dorsal

${ }^{1}$ Les ganglions de la base sont un ensemble de structures nerveuses (noyaux) enfouies profondément sous le cortex et interconnectées entres elles.

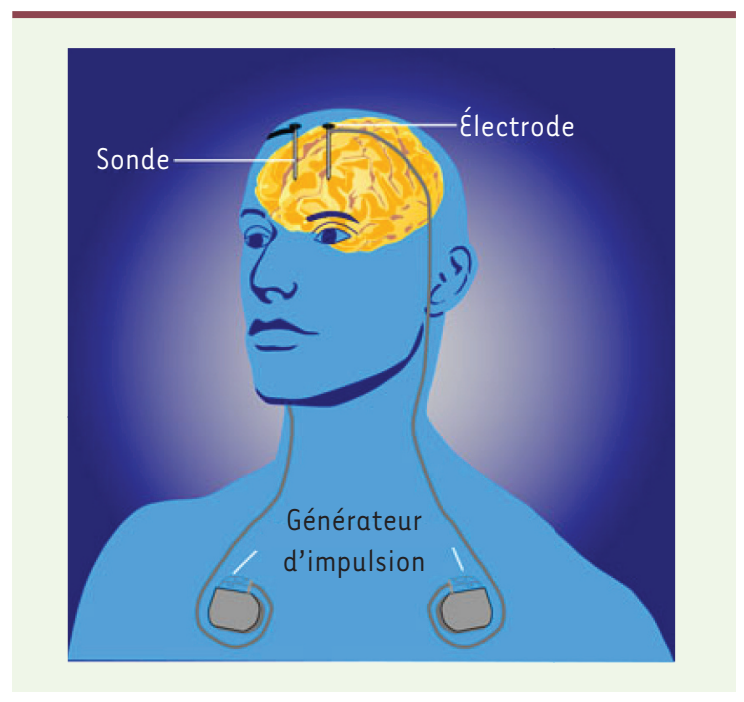

Figure 1. Mise en place de la stimulation cérébrale profonde chez l'homme (@ Creative commons Wikimedia).

lorsque l'usage devient plus intensif, c'est-à-dire à un moment où l'utilisation compulsive de drogue peut émerger [3]. On peut donc se demander si le choix de cibler une structure qui n'est finalement pas spécifiquement mobilisée en situation pathologique se justifie et laisse augurer d'une efficacité satisfaisante de la manipulation de cette structure dans le traitement de désordres toxicomaniaques.

2. Compte tenu de la participation du NAc à de nombreux processus comportementaux, il est peu probable que les manipulations de cette structure n'entraînent pas de perturbations comportementales autres que celles qui ont été ciblées.

Ainsi, il n'est pas surprenant de constater des revers après l'ablation thérapeutique du NAc. Réintroduite en Chine entre 2000 et 2004 pour le traitement de la dépendance à l'héroïne et à l'alcool, cette procédure a montré des résultats hétérogènes. Le taux d'abstinence 4 ans après l'intervention varie en effet entre $20 \%$ et $75 \%$ de succès, et de graves effets secondaires comme le manque de concentration, une mauvaise mémoire à court terme, des changements dans le désir sexuel, et une perte plus ou moins accentuée d'intérêt, ont été observés [4].

Une stimulation profonde de la région du NAc a bien entraîné un arrêt de l'usage de tabac ou d'alcool chez des patients souffrant de TOC, d'anxiété, de dépression ou du syndrome de Gilles de La Tourette [5-7]. Cependant, ces cas restent pour la plupart isolés, extrêmement hétérogènes et l'usage de drogue n'était, chez ces patients, qu'un syndrome associé. Rappelons de plus que la stimulation profonde du NAc peut être associée à des symptômes limbiques imprévisibles tels que la 


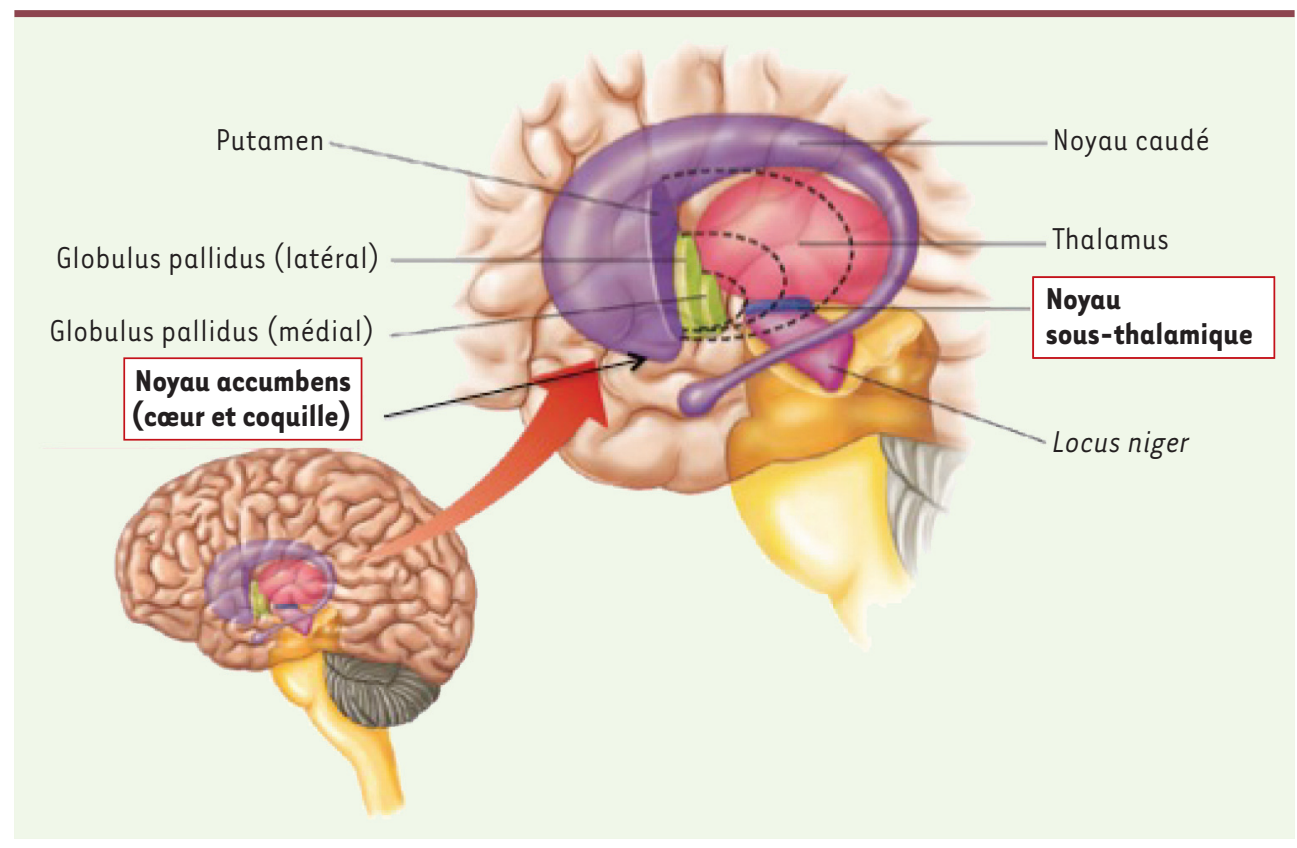

Figure 2. Localisation des structures des ganglions de la base auxquelles appartiennent les deux cibles privilégiées actuellement pour l'application de la stimulation cérébrale profonde dans le contexte des addictions, le noyau accumbens et le noyau sous-thalamique (ㄷ) Image Marc Savasta, Grenoble).

portement d'autoadministration d'héroïne [18].

Chez le rat, la stimulation profonde du NAc (core ou shell) peut réduire la consommation d'alcool [19]. La stimulation profonde de la partie dorso-

manie et la dépression $[5,8]$, une augmentation du degré d'impulsivité [9] et des oublis ou des difficultés à trouver ses mots [10]. Quelques groupes cliniques envisagent malgré tout, s'ils ne l'évaluent déjà, I'application en première intention de la stimulation profonde du NAc dans le traitement de la dépendance, soit à l'alcool, soit à l'héroïne [11]. Aux Pays-Bas, la technique semble donner de bons résultats chez un patient dépendant à l'héroïne, qui est en rémission après 6 mois [12]. En Chine, un autre patient est toujours abstinent après 6 ans [13] et en Allemagne, deux sont en rémission après 2 ans [14]. Toujours en Allemagne, un patient dépendant à l'alcool était toujours abstinent après 12 mois de stimulation profonde du NAc [15]. Au sein d'une cohorte de trois patients, une diminution de la consommation d'alcool a pu être observée chez 1 patient, et une abstinence complète persistait même après 15 mois chez 2 patients [16]. Mais, ces études récentes ont été réalisées sur des échantillons restreints de patients, sans que les divers aspects de leur motivation soient analysés, ce qui limite donc les conclusions quant à l'efficacité du traitement. Ces études ne permettent pas non plus de mettre en évidence les effets potentiellement délétères de ce type d'approche. Les réserves que nous émettons ne pourront être levées, ou au contraire confirmées, que par d'autres études; elles sont toutefois difficiles à multiplier pour des raisons éthiques.

Chez le rat, le NAc n'est pas une structure homogène et peut être dissocié en deux compartiments fonctionnels, la partie ventro-latérale (core) et la partie dorso-médiane (shell). Ces deux parties sont mal caractérisées chez l'homme [17]. Le faible nombre de patients traités ne permet pas une étude détaillée des effets comportementaux en fonction du site ciblé. A contrario, les études précliniques se sont intéressées à ces deux compartiments. La stimulation profonde à haute fréquence de la région core du NAc, de même que sa lésion ou son inactivation pharmacologique, réduisent la motivation à rechercher les effets de la morphine [10] et diminuent le rétablissement du com- médiane (shell), comme son inactivation pharmacologique, réduisent également la préférence et la consommation d'alcool $[20,21]$. Elle peut aussi réduire le comportement de recherche de cocaïne sans affecter la recherche de nourriture $[22,23]$. Cependant, certains auteurs ont mis en évidence une diminution de motivation pour la nourriture induite par la stimulation profonde de cette région du NAc [24]. Récemment, une étude a montré, dans d'autres conditions, que la stimulation profonde du NAc réduisait la recherche de cocaïne et celle de nourriture [25]. Plus récemment, il a été observé que les effets d'une stimulation profonde du NAc appliquée à basse fréquence pouvaient s'opposer aux mécanismes cellulaires associés à la sensibilisation induite par des injections répétées de cocaïne [26]. Toutefois, les modifications comportementales (sensibilisation motrice aux effets de la cocaïne) sont induites par quelques injections passives de cocaïne. Ceci ne reflète ainsi ni les conditions dans lesquelles le comportement toxicomaniaque émerge ni les altérations comportementales proprement pathologiques de ce trouble. Cette étude remarquable dissèque néanmoins les mécanismes induits par la stimulation profonde en utilisant $(\rightarrow)$ Voir la Synthèse de G.P. Dugué et L. Tricoire, $m / s \mathrm{n}^{\circ} 3$, mars 2015, page 291 l'optogénétique [46] $(\rightarrow)$ mais il est probable que ces mécanismes ne soient pas généralisables à la stimulation profonde appliquée à d'autres structures cérébrales, et notamment au noyau sous-thalamique (NST). À ce jour, les études conduites chez le rat, associées aux quelques cas cliniques observés, ne permettent donc pas de conclure avec certitude à un effet bénéfique 
de la stimulation cérébrale profonde. $\varepsilon$ n revanche, elles semblent en partie confirmer les risques d'une diminution de toute autre forme de motivation chez le toxicomane.

\section{Stimulation profonde du noyau sous-thalamique}

La stimulation cérébrale profonde du noyau sous-thalamique a d'abord été utilisée chez des patients parkinsoniens [1]. Plus récemment, elle a été appliquée à des patients souffrant de TOC [27, 45]. Chez ces deux types de patients, aucun effet de la stimulation profonde du noyau sousthalamique sur leur consommation de tabac ou d'alcool n'a été rapporté. Cependant, il y a très peu de parkinsoniens qui soient fumeurs, voire alcooliques. La stimulation profonde du noyau sous-thalamique n'a pas encore été testée en première intention chez des sujets toxicomanes. En revanche, de manière intéressante, pour certains patients atteints de la maladie de Parkinson qui développent une prise compulsive et une dépendance à la L-DOPA en dépit de sévères effets secondaires (on parle alors de syndrome de dérégulation dopaminergique), la stimulation profonde du noyau sousthalamique a montré des effets bénéfiques sur leur addiction à la L-DOPA [28-30].

Pourquoi cette structure, initialement considérée comme principalement impliquée dans les processus moteurs, pourrait-elle constituer une bonne cible pour le traitement de certaines addictions? C'est grâce à l'observation des effets secondaires de la stimulation profonde chez des patients parkinsoniens, et à des travaux réalisés chez l'animal, que le noyau sous-thalamique a été récemment impliqué dans d'autres processus que la motricité, comme les processus de motivation, cognitifs et limbiques. Ainsi, des études d'enregistrement de l'activité des neurones du noyau sous-thalamique, réalisées chez le rat et le singe, ont permis de mettre en évidence des changements d'activité en relation avec I'anticipation ou la présentation de récompenses diverses [31-33]. Chez l'homme également, un changement du niveau d'activité des neurones du noyau sous-thalamique a été observé par imagerie fonctionnelle chez des sujets héroïnomanes auxquels étaient présentées des images associées à la prise d'héroïne [34]. De plus, nous avons montré que l'inactivation du noyau sous-thalamique, par lésion ou stimulation profonde, diminue la motivation pour la cocaïne tout en augmentant celle pour une récompense naturelle, comme la nourriture ou le sucre $[35,36]$ (Figure 3A-B).

Les effets que nous avons observés lors d'une stimulation profonde du noyau sous-thalamique, diminution de motivation pour la drogue accompagnée d'une augmentation de motivation pour une récompense naturelle, sont en accord avec l'objectif recherché pour soigner un individu toxicomane: réduire sa motivation pour l'objet de l'addiction tout en restituant, ou en maintenant, sa motivation pour le reste. Ils font du noyau sous-thalamique une cible potentielle d'un traitement de la toxicomanie par stimulation profonde [37]. II faut toutefois prendre en considération l'observation d'une augmentation de la motivation pour un «renforçateur » naturel ${ }^{2}$ pour prévenir

${ }^{2}$ Le renforcement se définit comme l'augmentation de la force d'une réaction par la présentation d'un stimulus adéquat. II a souvent la forme d'une récompense. Un renforçateur primaire est quelque chose qui va satisfaire un besoin biologique ou un besoin physique de manière immédiate, sans aucun apprentissage antérieur. Les renforçateurs primaires sont naturels, c'est-à-dire non acquis. Par exemple, la nourriture, le sexe, etc. d'éventuels effets secondaires qui pourraient conduire à un dérèglement, de la prise alimentaire par exemple. Cependant, chez le rat, aucun excès de consommation alimentaire n'a été observé lorsque l'animal a un accès illimité à la nourriture [38].

Nous avons récemment montré que les résultats observés avec la cocaïne pouvaient être reproduits avec d'autres substances telles que l'héroïne (Slone-Murphy et al., en préparation), voire l'alcool dans une certaine mesure (Pelloux et Baunez, en préparation). D'autres auteurs ont également montré que l'administration du neuropeptide ocytocine directement dans le noyau sous-thalamique permettait de réduire la motivation à rechercher les effets de récompense qu'induit la métamphétamine [39].

Avant d'envisager de tester notre hypothèse chez le toxicomane, l'efficacité de la stimulation profonde du noyau sous-thalamique doit être évaluée dans d'autres modèles permettant de modéliser au mieux les troubles de l'addiction. Un modèle de perte de contrôle de la prise de drogue a été développé par Serge Ahmed et Georges Koob [40]. Dans ce modèle dans lequel un accès prolongé à la drogue est donné, nos résultats montrent que la stimulation profonde du noyau sousthalamique est efficace pour empêcher l'escalade de prise de drogue chez les animaux (Figure 3C) (Pelloux, et al., en préparation)

Il est toutefois intéressant de noter que l'inactivation du noyau sous-thalamique semble exercer son effet sur la motivation par un émoussement hédonique en réduisant les réponses affectives induites par du sucre ou de la quinine par exemple [41]. Ces résultats sont à rapprocher de certaines observations concernant les changements émotionnels observés chez les patients parkinsoniens soumis à une stimulation profonde du noyau sous-thalamique [42].

\section{Conclusion}

Cette observation d'un changement émotionnel associé à la stimulation profonde du noyau sous-thalamique chez des patients parkinsoniens témoigne de la difficulté qu'il y a à choisir une cible pour la stimulation profonde sans prendre le risque d'être confronté à des effets secondaires plus ou moins prévisibles. Comme dans le cas de la maladie de Parkinson, le choix ne peut se faire qu'en évaluant le rapport coût/bénéfice pour le patient. Dans le cas des addictions, malgré l'engouement des chercheurs pour évaluer plusieurs cibles cérébrales chez l'animal, l'hypothèse d'une application clinique se heurtera probablement à la difficulté du recrutement des patients. 

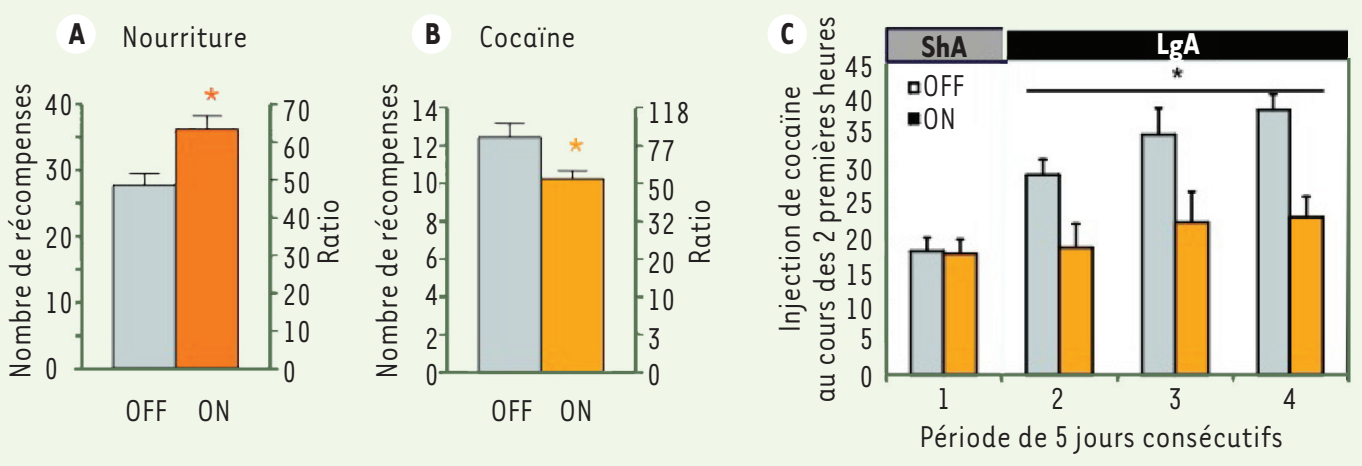

Figure 3. Effets de la stimulation cérébrale profonde du noyau sous-thalamique chez le rat. A. Dans un test évaluant la motivation à fournir un effort pour obtenir une récompense alimentaire sucrée. $B$. Dans le même test évaluant l'effort fourni pour s'auto-administrer de la cocaïne. C. Dans un modèle de perte de contrôle de la consommation de cocaïne : le modèle d'escalade. On voit en $\boldsymbol{A}$ et $\boldsymbol{B}$ que la stimulation profonde (noté $\mathrm{ON}$ ) induit une augmentation de motivation pour la nourriture sucrée, alors qu'elle induit une diminution pour la cocaïne. $C$. les animaux ont d'abord eu un accès court à la cocaïne (2h/jour pendant 5 jours, noté ShA), puis ils ont eu un accès prolongé pendant 15 jours consécutifs (6h/ jour, 3 blocs de 5 jours; noté LgA). On voit que dans les conditions d'accès prolongé, les animaux non stimulés (noté OFF) augmentent progressivement leur prise de cocaïne, alors que les animaux stimulés (noté $\mathrm{ON}$ ) maintiennent un niveau stable de consommation. La stimulation cérébrale profonde du noyau sous-thalamique a donc un impact sur le basculement de l'addiction vers la recherche compulsive de la drogue (A et $B: \mathrm{d}$ 'après [36] ; C : Pelloux et al., en préparation).

La question de la justification de l'application d'une technique invasive chez des toxicomanes doit être évidemment posée. Étant donné le peu de solutions thérapeutiques offertes à ces patients, on peut penser que la stimulation profonde puisse être considérée.

Comme nous l'avons évoqué ci-dessus, le choix de la région cérébrale ciblée, comme les modalités de stimulation (en continu ou au moment du craving ${ }^{3}$ pour la drogue) restent à déterminer. Ils pourraient varier en fonction du type de dépendance. Toutes ces interrogations font l'objet des recherches actuelles dans ce domaine. $\diamond$

\section{SUMMARY}

\section{Surgical strategy to rescue the addicts}

Since its successful application for the treatment of neurological disorders, deep brain stimulation (DBS) is currently also applied for the treatment of psychiatric disorders such as obsessive compulsive disorders or depression. DBS is being considered, or even applied, as a treatment for certain forms of addiction. We review here the cerebral structures aimed for such a strategy and discuss their respective positive and negative aspects. $\diamond$

\section{LIENS D'INTÉRÊT}

Les auteurs déclarent n'avoir aucun lien d'intérêt concernant les données publiées dans cet article.

\section{RÉFÉRENCES}

1. Limousin P, Pollak P, Benazzouz A, et al. Effect of Parkinsonian signs and symptoms of bilateral subthalamic nucleus stimulation. Lancet $1995 ; 345$ : 91-5.

2. Ersche KD, Barnes A, Jones PS, et al. Abnormal structure of frontostriatal brain systems is associated with aspects of impulsivity and compulsivity in cocaine dependence. Brain 2011 ; $134: 2013-24$.

3. Porrino LJ, Smith HR, Nader MA, Beveridge TJ. The effects of cocaine: $a$ shifting target over the course of addiction. Prog Neuropsychopharmacol Biol Psychiatry $2007 ; 31: 1593-600$.

4. Li N, Wang J, Wang XL, et al. Nucleus accumbens surgery for addiction. World Neurosurg $2013 ; 80$ : S28.e9-19.

5. Kuhn J, Lenartz D, Huff W, et al. Remission of alcohol dependency following deep brain stimulation of the nucleus accumbens : valuable therapeutic implications? J Neurol Neurosurg Psychiatry 2007 ; 78 : 1152-3.

6. Kuhn J, Bauer R, Pohl S, et al. Observations on unaided smoking cessation after deep brain stimulation of the nucleus accumbens. Eur Addict Res 2009 ; $15: 196-201$.

7. Mantione M, van de Brink W, Schuurman PR, Denys D. Smoking cessation and weight loss after chronic deep brain stimulation of the nucleus accumbens: therapeutic and research implications: case report. Neurosurgery $2010 ; 66$ : E218.

8. Saleh C, Okun MS. Clinical review of deep brain stimulation and its effects on limbic basal ganglia circuitry. Front Biosci 2008 ; 13 : 5708-31.

9. Luigjes J, Mantione M, van den Brink W, et al. Deep brain stimulation increases impulsivity in two patients with obsessive-compulsive disorder. Int Clin Psychopharmacol $2011 ; 26: 338-40$.

10. Liu HY, Jin J, Tang JS, et al. Chronic deep brain stimulation in the rat nucleus accumbens and its effect on morphine reinforcement. Addict Biol 2008; 13 : 40-6.

11. Stelten BM, Noblesse LH, Ackermans L, et al. The neurosurgical treatment of addiction. Neurosurg Focus $2008 ; 25: \varepsilon 5$.

12. Valencia-Alfonso C $\varepsilon$, Luigjes J, Smolders $R$, et al. Effective deep brain stimulation in heroin addiction: a case report with complementary intracranial electroencephalogram. Biol Psychiatry 2012; 71 : e35-7.

13. Zhou H, Xu J, Jiang J. Deep brain stimulation of nucleus accumbens on heroin-seeking behaviors: a case report. Biol Psychiatry 2011 ; 69 : e4l-2. 


\section{RÉFÉRENCES}

14. Kuhn J, Möller M, Treppmann JF, et al. Deep brain stimulation of the nucleus accumbens and its usefulness in severe opioid addiction. Mol Psychiatry $2014 ; 19$ : 145-6.

15. Kuhn J, Gründler TO, Bauer R, et al. Successful deep brain stimulation of the nucleus accumbens in severe alcohol dependence is associated with changed performance monitoring. Addict Biol $2011 ; 16: 620-3$

16. Müller UJ, Sturm V, Voges J, et al. Successful treatment of chronic resistant alcoholism by deep brain stimulation of nucleus accumbens: first experience with three cases. Pharmacopsychiatry $2009 ; 42: 288-91$

17. Heimer L. Basal forebrain in the context of schizophrenia. Brain Res Brain Res Rev $2000 ; 31: 205-$ 35.

18. Guo L, Zhou H, Wang R, et al. DBS of nucleus accumbens on heroin seeking behaviors in selfadministering rats. Drug Alcohol Depend $2013 ; 129: 70-81$.

19. Knapp CM, Tozier L, Pak A, et al. Deep brain stimulation of the nucleus accumbens reduces ethanol consumption in rats. Pharmacol Biochem Behav $2009 ; 92: 474-9$.

20. Henderson MB, Green Al, Bradford PS, et al. Deep brain stimulation of the nucleus accumbens reduces alcohol intake in alcohol-preferring rats. Neurosurg Focus $2010 ; 29: \varepsilon 12$.

21. Wilden JA, Qing KY, Hauser SR, et al. Reduced ethanol consumption by alcohol-preferring (P) rats following pharmacological silencing and deep brain stimulation of the nucleus accumbens shell. $J$ Neurosurg $2014 ; 120: 997-1005$.

22. Vassoler FM, Schmidt HD, Gerard ME, et al. Deep brain stimulation of the nucleus accumbens shel attenuates cocaine priming-induced reinstatement of drug seeking in rats. J Neurosci $2008 ; 28$ : 8735-9.

23. Vassoler FM, White SL, Hopkins TJ, et al. Deep brain stimulation of the nucleus accumbens shell attenuates cocaine reinstatement through local and antidromic activation. J Neurosci 2013 ; 33 14446-54.

24. Van der Plasse G, Schrama R, van Seters SP, et al. Deep brain stimulation reveals a dissociation of consummatory and motivated behaviour in the medial and lateral nucleus accumbens shell of the rat. PLoS One $2012 ; 7$ : e33455.

25. Guercio LA, Schmidt HD, Pierce RC. Deep brain stimulation of the nucleus accumbens shell attenuates cue-induced reinstatement of both cocaine and sucrose seeking in rats. Behav Brain Res 2014 ; 281C : 125-30

26. Creed M, Pascoli VJ, Lüscher C. Refining deep brain stimulation to emulate optogenetic treatment of synaptic pathology. Science $2015 ; 347: 659-64$.

27. Mallet $L$, Polosan $M$, Jaafari N, et al. Subthalamic nucleus stimulation in severe obsessivecompulsive disorder. $N$ Engl J Med 2008 ; 359 : 2121-34

28. Witjas T, Baunez C, Henry JM, et al. Addiction in Parkinson's disease: impact of subthalamic nucleus deep brain stimulation. Mov Disord 2005 ; 20 : 1052-5.

29. Lhommee $\varepsilon$, Klinger $H$, Thobois $S$, et al. Subthalamic stimulation in Parkinson's disease: restoring the balance of motivated behaviours. Brain $2012 ; 135: 1463-77$.

30. Eusebio A, Witjas T, Cohen J, et al. Subthalamic nucleus stimulation and compulsive use of dopaminergic medication in Parkinson's disease. J Neurol Neurosurg Psychiatry $2013 ; 84$ : 868-74.

31. Lardeux S, Pernaud R, Paleressompoulle D, Baunez C. Beyond the reward pathway: coding of the reward salience and error in the rat subthalamic nucleus. J Neurophysiol $2009 ; 102$ : 2526-37.
32. Lardeux S, Paleressompoulle D, Pernaud R, et al. Different populations of subthalamic neurons encode cocaine and sucrose reward and predict error. J Neurophysiol 2013 ; $110: 1497-510$.

33. Darbaky Y, Baunez C, Arecchi P, et al. Reward-related neuronal activity in the subthalamic nucleus of the monkey. Neuroreport $2005 ; 16$ : 1241-4.

34. Zijlstra F, Veltman DJ, Booij J, et al. Neurobiological substrates of cueelicited craving and anhedonia in recently abstinent opioid-dependent males. Drug Alcohol Depend 2009; 99 : 183-92.

35. Baunez C, Dias C, Cador M, Amalric M. The subthalamic nucleus exerts opposite control on cocaine and natura rewards. Nat Neurosci $2005 ; 8$ : $484-9$

36. Rouaud T, Lardeux S, Panayotis N, et al. Reducing the desire for cocaine with subthalamic nucleus deep brain stimulation. Proc Natl Acad Sci USA 2010 ; $107: 1196-200$

37. Pelloux $y$, Baunez C. Deep brain stimulation for addiction: why the subthalamic nucleus should be favored. Curr Opin Neurobiol $2013 ; 23$ $713-20$.

38. Baunez C, Amalric M, Robbins TW. Enhanced food-related motivation after bilateral lesions of the subthalamic nucleus. J Neurosci $2002 ; 22$ : 562-8.

39. Baracz SJ, Rourke PI, Pardey MC, et al. Oxytocin directly administered into the nucleus accumbens core or subthalamic nucleus attenuates methamphetamine-induced conditioned place preference. Behav Brain Res $2012 ; 228: 185-93$

40. Ahmed SH, Koob GF. Transition from moderate to excessive drug intake: change in hedonic set point. Science $1998 ; 282: 298-300$.

41. Pelloux Y, Meffre J, Giorla $\varepsilon$, Baunez C. The subthalamic nucleus keeps you high on emotion: behavioral consequences of its inactivation. Frontiers Behav Neurosci $2014 ; 8: 414$

42. Péron J, Frühholz $S$, Vérin $M$, Grandjean D. Subthalamic nucleus: a key structure for emotional component synchronization in humans. Neurosci Biobehav Rev 2013 ; 37 : 358-73.

43. Ramoz N, Gorwood P. Les addictions sous l'angle de la génétique. Med Sci (Paris) $2015 ; 31: 432-8$

44. Arango-Lievano M, KaplittMG. Comorbidité entre la dépression et l'addiction: vers une cible moléculaire commune? Med Sci (Paris) 2015 $31: 546-50$.

45. Alves dos Santos JF, Mallet L. Le trouble obsessionnel compulsif. Med Sci (Paris) $2013 ; 29: 1111-6$

46. Dugué GP, Tricoire L. Principes et applications de l'optogénétique en neuroscience. Med Sci (Paris) 2015 ; 31 : 291-303.

\section{TIRÉS À PART}

C. Baunez

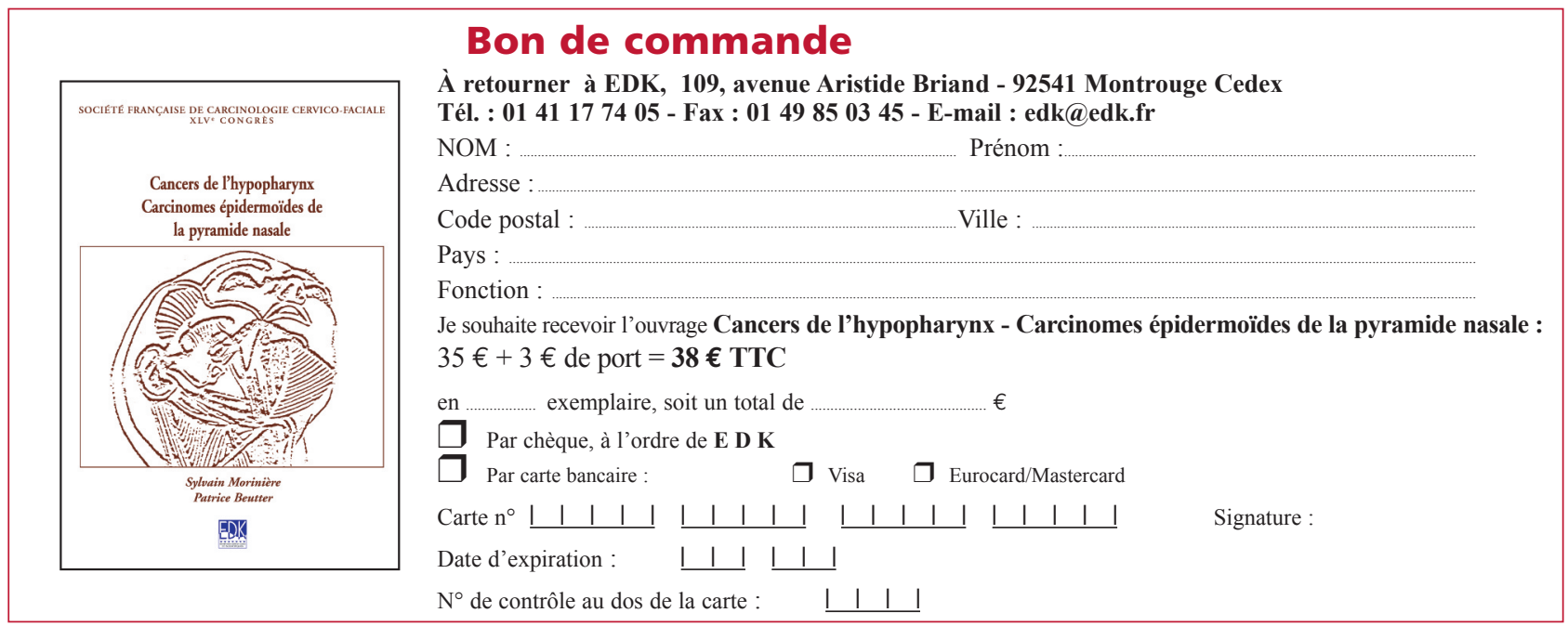

